\title{
A EFICÁCIA DA UTILIZAÇÃO DE UMA PALMILHA ORTOPÉDICA NA DISTRIBUIÇÃO DA PRESSÃO PLANTAR E NO QUADRO ÁLGICO DE PACIENTES COM FASCITE PLANTAR
}

\author{
Ramiro Marques Inchauspe \\ Pablo Morales Barbian
}

\begin{abstract}
RESUMO A fascite plantar é uma inflamação uni ou bilateral, decorrentes de micro traumatismos repetitivos na origem da fáscia plantar sobre a tuberosidade medial do calcâneo tendo em vista o difícil tratamento desta patologia, a baropodometria é utilizada na avaliação das disfunções biomecânicas dos pés, principalmente no controle da distribuição da pressão plantar. O trabalho teve como objetivo verificar a influência da utilização de uma órtese no comportamento da distribuição da pressão plantar durante a marcha e no quadro álgico de pacientes com fascite plantar. A população estudada constou de vinte voluntários da faixa etária entre 23 e 58 anos, sendo dez do Grupo Controle GC (4 homens e 6 mulheres) e dez no Grupo Fascite GF ( 4 homens e 6 mulheres). Foi questionado no grupo fascite qual a localização e intensidade da dor numa escala de zero a dez. Foi realizado uma avaliação da distribuição da pressão plantar foi utilizada uma plataforma sensorizada do sistema Miletrix. Para as médias da intensidade da dor entre a avaliação Préintervenção e Pós-intervenção foram encontradas diferenças significativas ( $t$ = $10,062, p=0,000$ ), porém as variáveis espaço-temporais analisadas nos pés direito e esquerdo foram: comprimento do passo, cadência, largura do passo, ângulo de progressão do pé, ângulo do eixo do pé e velocidade. Após a avaliação inicial, foi confeccionada um par de órteses para o GF que foram instruídos a utilizar a órtese durante 54 dias ininterruptos, após este período foi realizada uma reavaliação utilizando os mesmos procedimentos da anterior. Foram observadas diferenças estatisticamente significativas entre o grupo GC e o GF para as variáveis pressão plantar máxima e pressão plantar média, para o pé direito. No pé esquerdo não foram observadas diferenças significativas. Para as demais variáveis (tempo de contato e área de contato) não foram observadas diferenças significativas entre o GC e o GF. Como resultado para o GF não foram
\end{abstract}


observadas diferenças significativas para as variáveis da distribuição da pressão plantar entre a avaliação pré-intervenção (uso da órtese) e pós-intervenção. Não foram encontrada diferenças estatisticamente significativas entre o GF e o GC para nenhuma das variáveis espaço-temporais. Também não foram encontradas diferenças significativas para o GF entre avaliação pré-intervenção e avaliação pós-intervenção. $\mathrm{O}$ uso da órtese provocou uma melhora no quadro álgico dos pacientes com fascite plantar, porém não provocou alterações significativas no comportamento da distribuição da pressão plantar e das variáveis espaçotemporais.

Palavras Chave: Fascite Plantar. Escala de dor. Pressão plantar. Espaçotemporais. 
ABSTRACT The plantar fasciitis is an inflammation of one or both sides, resulting from repetitive micro trauma to the origin of the plantar fascia on the medial calcaneal tuberosity in view of the difficult treatment of this pathology, baropodometry is used in the assessment of biomechanical disorders of the feet, especially in control of plantar pressure distribution. The study aimed to verify the influence of the use of a stent in the behavior of the plantar pressure distribution during gait and pain status of patients with plantar fasciitis. The study population consisted of twenty volunteers aged between 23 and 58 years, ten of the GC control group ( 4 men and 6 women) and ten in Group fasciitis GF (4 men and 6 women). Was asked in the fasciitis group which the location and intensity of pain on a scale of zero through ten. An evaluation of plantar pressure distribution sensorizada one platform Miletrix system was used was performed. For the average pain intensity between the assessment pre-intervention and postintervention significant differences $(t=10.062, p=0.000)$ were found, but the spatio-temporal variables analyzed in the right and left feet: stride length, cadence, step width angle of progression of the foot, the foot shaft angle and speed. After the initial assessment, was made a pair of orthotics for the GF who were instructed to wear the brace for 54 uninterrupted days, after this period a reassessment was performed using the same procedures as before. Statistically significant differences between the control group and the GF were observed for the variables maximum plantar pressure and plantar pressure medium, to the right foot. Left foot, no significant differences were observed. For the other variables (contact time and contact area) no significant differences between the GC and the GF were observed. As a result for the GF no significant differences for the variables of plantar pressure distribution between the pre-intervention (orthosis) and post-intervention were observed. No statistically significant differences were found between the GF and the GC for the variables spatiotemporal. Also no significant differences were found between the GF preintervention assessment and post-intervention assessment. The orthosis caused an improvement in pain symptoms in patients with plantar fasciitis, but no significant changes in the behavior of the plantar pressure distribution and spatiotemporal variables.

Keywords: Plantar Fasciitis. Pain scale. Plantar pressure. Space-time. 


\section{INTRODUÇÃO}

A biomecânica do pé e do tornozelo é complexa e intrinsecamente associadas entre si. O pé é parte mecânica integrante da extremidade inferior necessária para o desenvolvimento de uma marcha suave e estável. O tornozelo realiza a transferência das cargas da extremidade inferior para o pé e influencia intimamente na orientação do pé com o solo (NORDIN e FRANKEL, 2003). Os autores ainda colocam o complexo do pé e tornozelo além de agir como uma estrutura de suporte capaz de resistir às cargas repetitivas múltiplas do peso corporal, deve também ajustar-se às diferentes superfícies do solo e às variações da velocidade durante a locomoção. Qualquer mudança patológica nas estruturas ou nos movimentos do pé e do tornozelo, embora sutil, pode ter um profundo impacto nas suas funções de absorção dos impactos, propulsiva e estabilizadora.

As alterações plantares podem ser responsáveis por desequilíbrios posturais, assim como os pés se ajustam aos desequilíbrios vindos de estruturas suprajacentes. Por ser o suporte final do sistema postural, os pés precisam se adaptar às irregularidades transferidas do corpo e das superfícies externas (BRICOTT, 1999).

Dentre as diversas alterações plantares que podem ocorrer, de acordo com Zanon et al. (2006), os estudos destacam que a fascite plantar é uma das alterações plantares que pode atingir em torno de $10 \%$ da população, em algum momento da vida, e as mulheres obesas na idade do climatério são as mais afetadas. A fascite plantar é considerada uma síndrome degenerativa, sendo caracterizada por uma inflamação, uni ou bilateral, que ocorre por microtraumatismos de repetição na origem da fáscia plantar, sobre a tuberosidade medial do calcâneo. Apresenta como sintomatologia a dor localizada na face plantar do calcâneo, podendo se espalhar ao longo da fáscia plantar (STARKEY e RYAN, 2001). Os sintomas de dor são mais evidentes pela manhã, ao dar o primeiro passo, ao se levantar da cama, ou após um período de repouso. A claudicação é comum para evitar o apoio do calcanhar no chão (WILLIAMS e WILKINS, 1996).

De acordo com Metzker (2012) a fascite plantar está relacionada com alterações anatômicas e/ou biomecânicas e suas causas podem estar 
associadas à diferença no comprimento dos membros inferiores, a pronação excessiva da articulação subtalar, a falta de flexibilidade do arco longitudinal, a rigidez muscular da panturrilha e o uso constante de calçados inadequados (PRENTICE, 2002 apud METZKER, 2012).

O diagnóstico de fascite plantar é feito apenas através da história clínica e do exame objetivo, sendo que a radiografia revela apenas um esporão do calcâneo e a ecografia mostra sinais inflamatórios, ao passo que a ressonância magnética nuclear mostra sinais semelhantes à tendinose na inserção no calcâneo. Os programas de tratamento da fascite plantar podem ser realizado através do tratamento conservador, que é feito através de alongamentos, gelo, eletroterapia, anti-inflamatórios, infiltrações e órteses (palmilhas ortopédicas), e através do tratamento cirúrgico (HEBERT e XAVIER, 2003). Da mesma forma, Ferreira (2014) destaca que o diagnóstico é essencialmente clínico e tem como base a história do paciente e o exame físico. Destaca também que exames complementares laboratoriais e de imagem podem ser úteis no diagnóstico diferencial da fascite plantar. Como tratamentos destaca o tratamento conservador, que preconiza um programa domiciliar de exercícios de alongamento da fáscia plantar, apresentando taxa de melhora do paciente em torno de $90 \%$, e o tratamento cirúrgico, que somente é indicado quando o tratamento conservador não apresenta melhora significativa dos sintomas de dor.

Para o diagnóstico da fascite plantar pode também ser utilizada a avaliação dinâmica da distribuição da pressão plantar, que fornece informações sobre a estrutura e a função dos pés, assim como informações sobre as alterações e controle postural do corpo, contribuindo para identificar condições saudáveis ou patológicas. A mensuração da pressão plantar pode fornecer informações sobre os padrões de distribuição de pressão e da força, tanto em postura estática como dinâmica (MANFIO et al., 1999). A avaliação da distribuição da pressão também pode ser conhecida na área clínica como baropodometria, que consiste na medição das pressões plantares dos pés, sendo muito utilizada em pesquisas sobre alterações biomecânicas e alterações motoras que comprometem a marcha (ALVAREZ et al., 2008).

A análise dos padrões biomecânicos da marcha tornou-se uma ferramenta importante nas últimas décadas, favorecendo estudos sobre o 
comportamento ósseo, muscular e articular para a prevenção e a reabilitação da perda ou da dificuldade de deambulação (BOVI et al., 2011). O estudo do comportamento e da função do complexo do pé facilita o desenvolvimento de tratamentos e métodos preventivos das alterações patológicas que dificultam a marcha normal (MICKLE et al., 2011). De acordo com Stebbins et al. (2005) e Nazario et al. (2010) a avaliação da distribuição de pressão plantar é utilizada na avaliação de problemas clínicos dos pés, anormalidades e pós-operatório, sendo considerado um método seguro e pode contribuir para o diagnóstico e para definição do tratamento.

Segundo Rosebaum e Becker (1997) e Orlin e Thomas (2000), os pés por serem a base de sustentação podem contribuir para as alterações posturais. Estas alterações posturais que são decorrentes do apoio plantar, podem ser minimizadas com a utilização de órteses, como palmilhas, sendo indispensável para complementar o tratamento. Alguns estudos (JANNINK et al., 2006; HODGE et al., 1999) abordam a importância de palmilhas ortopédicas, também conhecidas como palmilhas funcionais, para a melhora da distribuição de pressão plantar. $\mathrm{O}$ uso de palmilhas para melhorar o alinhamento e o conforto dos pés é antigo e dentro do grupo das órteses, são os tratamentos mais comum na patologia dos pés, contribuindo para o alívio da dor e para a melhora clínica dos pacientes (MIYAZAKI e ANDRADE, 2010).

Com base nas colocações acima o objetivo deste estudo foi verificar a influência da utilização de uma órtese no comportamento da distribuição da pressão plantar durante a marcha e no quadro álgico de pacientes com fascite plantar.

\section{MÉTODO}

Foram convidados de maneira aleatória vinte voluntários, que foram divididos em dois grupos: Grupo Fascite Plantar (GF), que foi composto por 10 voluntários (4 homens e 6 mulheres) com diagnóstico clínico de fascite plantar, na faixa etária entre 23 e 58 anos (média de idade 35,8 \pm 12,1 anos), massa corporal 78,7 $\pm 20,5 \mathrm{Kg}$ e estatura $169,7 \pm 8,6 \mathrm{~cm}$, e o Grupo Controle (GC), composto por 10 voluntários (4 homens e 6 mulheres) sem histórico de fascite 
plantar, na faixa etária entre 21 e 53 anos (média de idade 31,8 \pm 10,4 anos), massa corporal 64,6 $\pm 12,8 \mathrm{Kg}$ e estatura de 168,9 \pm 9,2 cm.

Todos os voluntários assinaram do Termo de Consentimento Livre e Esclarecido (TCLE), em conformidade com a resolução $N^{\circ}$ 466, de 12 de dezembro de 2012, do Conselho Nacional de Saúde, aprovado pelo Comitê de Ética e Pesquisa (CEP) da Universidade FEEVALE, parecer $n^{\circ} 242.459$.

Este estudo é caracterizado como do tipo experimental de caráter descritivo, e adotou os seguintes critérios de inclusão: para o grupo fascite plantar (GF), assinatura do TCLE e voluntários na faixa etária de 18 a 59 anos de idade com diagnóstico clínico de fascite plantar, e, para o grupo controle (GC), assinatura do TCLE, voluntários na faixa etária de 18 a 59 anos de idade sem diagnostico de fascite plantar e sem alterações músculo-esquelético nos membros inferiores.

Os dados coletados serão tabulados e classificados para a realização da análise estatística do estudo através de um banco de dados em planilha

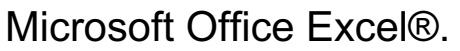

Foi utilizada estatística descritiva através de frequências absolutas (n) e relativas (\%), média e desvio-padrão. Para a análise da comparação antes e depois dos resultados foi utilizado o Teste de Pearson com nível de significância de $p \leq 0,05$.

Também foi utilizado uma analise descritiva, onde os dados serão descritos e interpretados individualmente.

Para a coleta de dados deste estudo foram utilizados os seguintes instrumentos: Questionário-Entrevista, para as avaliações das características demográficas dos participantes e avaliação da intensidade da dor, através da Escala de Dor Associada (CARVALHO e KOWACS, 2006), composta pelas escalas: Analógica Visual Numérica (EAV), Categórica de Avaliação (ECA) e Desabilitante Funcional (EDF), conforme descrito adaptado por Reichert (2012). Balança Digital e Estadiômetro, para avaliação antropométrica; Plataforma de pressão do sistema Miletrix (Diagnostic Suport), para avaliação da distribuição de pressão plantar durante a marcha descalça.

Após o recrutamento dos voluntários e a assinatura do TCLE, iniciou-se a coleta de dados, através do questionário-entrevista que abordou aspectos como, dados de identificação, investigação ortopédica, incidência de calosidades e 
deformações nos pés, avaliação da intensidade da dor e localização dos sintomas de dor. Para a avaliação da distribuição de pressão plantar durante a marcha descalça foi utilizada uma plataforma sensorizada do sistema Miletrix da marca DiagnosticSuport. O sistema Miletrix é composto por uma plataforma com 4800 sensores capacitivos, com resolução de 4 sensores $/ \mathrm{cm}^{2}$ e taxa de aquisição de $25 \mathrm{~Hz}$. A plataforma apresenta as seguintes dimensão: $120 \mathrm{~cm}$ de comprimento e $60 \mathrm{~cm}$ de largura. Os dados da distribuição de pressão plantar foram coletados e analisados pelo software do próprio sistema Miletrix, versão 1.0.0.26.

Para a avaliação da marcha descalça, de ambos os grupos (GF e GC), o indivíduo foi instruído a comparecer no local de coleta de dados com roupa adequada (bermuda e camiseta). A avaliação da marcha foi realizada com velocidade auto-selecionada, sendo que o participante deveria caminhar em uma passarela de $4,50 \mathrm{~m}$, estando a plataforma sensorizada instalada a $2 \mathrm{~m}$ do início da passarela. Foram coletadas cinco tentativas consideradas válidas, onde o primeiro contato com a plataforma foi realizado com o pé direito e o segundo contato com o pé esquerdo, estando ambos os pés sobre a área sensorizada da plataforma. Durante a avaliação da distribuição de pressão plantar os participantes não apresentaram alterações na marcha, como desequilíbrios, e o olhar foi direcionado para frente.

Após a avaliação inicial (Pré-intervenção), com base nos dados da avaliação da distribuição de pressão plantar de cada participante do grupo fascite plantar (GF), foi confeccionado um par de órteses de Etileno Acetato de Vinila (EVA), com densidades que podem variar de 20 a 50 SHORE A. Os participantes foram instruídos a utilizar a órtese durante 54 dias interruptos em média, e após o período experimental, foi realizada a reavaliação utilizando os mesmos procedimentos da pré-intervenção.

Assim, a coleta dos dados foi composta por duas avaliações: Préintervenção, avaliação realizada com os dois grupos (GF e GC) antes do período de intervenção (uso da órtese específica), sendo que os dados desta avaliação foram utilizados para a definição das características da órtese (palmilha) para os participantes do GF, e Pós-intervenção, avaliação realizada com o GF após o período de intervenção (a cerca de 54 dias). 


\section{RESULTADOS}

Conforme apresentado na Figura 1, quando os participantes do Grupo Fascite Plantar (GF) foram questionados sobre a intensidade da dor, conforme a Escala de Dor Associada (CARVALHO e KOWACS, 2006), observou-se que na avaliação Pré-intervenção (avaliação antes do uso da órtese) a média da intensidade da dor foi de 7,4 $\pm 2,0$ pontos e na avaliação Pós-intervenção (avaliação após o período de uso da órtese) a média foi de $1,4 \pm 1,3$ pontos. Para as médias da intensidade da dor entre a avaliação Pré-intervenção e Pósintervenção foram encontradas diferenças significativas $(t=10,062, p=0,000)$.

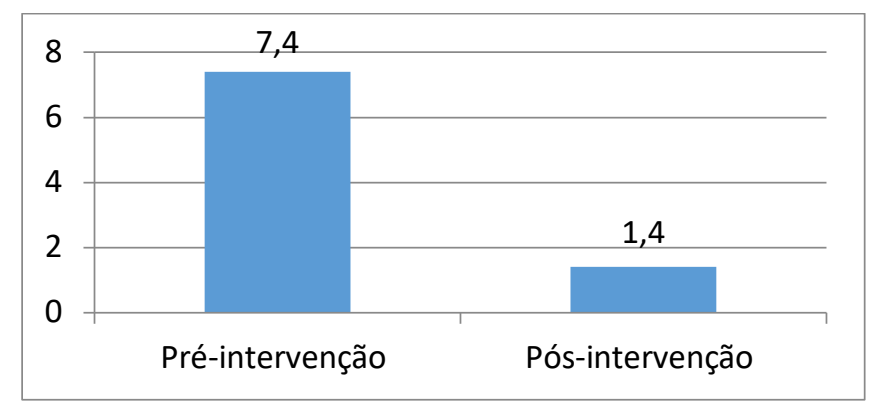

Figura 1 - Intensidade da dor entre a avaliação Pré-intervenção e Pósintervenção para o GF.

O GF relatou que à localização dos sintomas da dor ocorreram na região inferior do arco plantar para 50\% (5) dos participantes, na região inferior do calcanhar para 40\% (4) dos participantes e em ambas as regiões (inferior do arco plantar e do calcanhar) para 10\% (1) dos participantes. Os sintomas de dor foram observados no pé direito em $60 \%$ (6) dos participantes, no pé esquerdo em $30 \%$ (3) e bilateral em 10\% (1) dos participantes. Foram relatados sintomas de dor em outras regiões do corpo, como na articulação do joelho (30\%), na articulação do quadril $(10 \%)$ e na coluna $(10 \%)$.

Com relação ao período do dia, em que os sintomas de dor do GF eram mais intensos, observou-se que $80 \%$ (8) dos participantes relataram sintomas de dor no período da manhã, 10\% (1) no período da tarde e 10\% (1) relataram que não tinha um período definido, ou seja, a dor era constante durante todo o dia.

Para a avaliação de outro tipo de tratamento conservador; observou-se que $70 \%$ dos participantes realizavam tratamento farmacológico ou 
fisioterapêutico, 30\% não realizavam nenhum tipo de tratamento e nenhum dos participantes do GF utilizava palmilha ortopédica. 90\% (9) dos participantes do GF relataram que não sofreram fraturas membros inferiores e 10\% (1) relataram fraturas nos dedos dos pés, joelho e pelve. Verificou-se que 50\% (5) do GF relataram torções membros inferiores e 50\% (5) não relataram torções. Em relação a calosidades na região plantar observou-se que em 40\% (4) do GF ocorreram na região do retropé (1) e do antepé (3) e em 60\% (6) não apresentavam calosidades na região plantar. Para a prática de atividade física observou-se que $60 \%$ (6) do GF praticam atividade física regularmente e $40 \%$ (4) não praticam atividade física.

Para o Grupo Controle (GC) observou-se nenhum dos sujeitos utilizavam palmilhas ortopédica e 50\% (5) praticavam atividades físicas regularmente.

$\mathrm{Na}$ Tabela 1 foram apresentados os dados referentes à distribuição de pressão plantar para o grupo controle (GC) e para o grupo fascite plantar (GF), na fase pré-intervenção e pós-intervenção. Foram observadas diferenças estatisticamente significativas entre o grupo GC e o GF para as variáveis pressão plantar máxima e pressão plantar média, para o pé direito. No pé esquerdo não foram observadas diferenças significativas. Para as demais variáveis (tempo de contato e área de contato) não foram observadas diferenças significativas entre o GC e o GF.

Para o GF não foram observadas diferenças significativas para as variáveis da distribuição da pressão plantar entre a avaliação pré-intervenção (uso da órtese) e pós-intervenção.

Tabela 1 - Dados referentes a distribuição de pressão plantar.

\begin{tabular}{|c|c|c|c|c|c|c|}
\hline & \multirow{2}{*}{\multicolumn{2}{|c|}{$\begin{array}{c}\begin{array}{c}\text { Grupo Controle } \\
\text { (Média } \pm \text { DP) }\end{array} \\
\text { Pré-Intervenção }\end{array}$}} & \multicolumn{4}{|c|}{$\begin{array}{l}\text { Grupos Fascite Plantar } \\
\text { (Média } \pm \text { DP) }\end{array}$} \\
\hline & & & \multicolumn{2}{|c|}{ Pré-Intervenção } & \multicolumn{2}{|c|}{ Pós-Intervenção } \\
\hline Variável & $\begin{array}{c}\text { Pé } \\
\text { esquerdo }\end{array}$ & $\begin{array}{l}\text { Pé } \\
\text { direito }\end{array}$ & $\begin{array}{c}\text { Pé } \\
\text { esquerdo }\end{array}$ & $\begin{array}{l}\text { Pé } \\
\text { direito }\end{array}$ & $\begin{array}{c}\text { Pé } \\
\text { esquerdo }\end{array}$ & $\begin{array}{l}\text { Pé } \\
\text { direito }\end{array}$ \\
\hline Tempo de contato (s) & $\begin{array}{c}0,65 \\
(0,07)\end{array}$ & $\begin{array}{c}0,64 \\
(0,64)\end{array}$ & $\begin{array}{c}0,65 \\
(0,11)\end{array}$ & $\begin{array}{c}0,63 \\
(0,10)\end{array}$ & $\begin{array}{c}0,65 \\
(0,09)\end{array}$ & $\begin{array}{c}0,63 \\
(0,11)\end{array}$ \\
\hline $\begin{array}{l}\text { Área de contato } \\
\left(\mathrm{cm}^{2}\right)\end{array}$ & $\begin{array}{c}78,96 \\
(20,23) \\
\end{array}$ & $\begin{array}{l}98,98 \\
(18,55) \\
\end{array}$ & $\begin{array}{c}82,48 \\
(21,30) \\
\end{array}$ & $\begin{array}{c}89,86 \\
(24,11) \\
\end{array}$ & $\begin{array}{c}83,02 \\
(28,20) \\
\end{array}$ & $\begin{array}{c}88,59 \\
(25,09) \\
\end{array}$ \\
\hline $\begin{array}{l}\text { Pressão Plantar Máxima } \\
\qquad\left(\mathrm{g} / \mathrm{cm}^{2}\right)\end{array}$ & $\begin{array}{l}1241,33 \\
(176,67)\end{array}$ & $\begin{array}{c}1008,34 \\
* \\
(86,90) \\
\end{array}$ & $\begin{array}{l}1386,19 \\
(255,57)\end{array}$ & $\begin{array}{l}1173,61^{*} \\
(139,21)\end{array}$ & $\begin{array}{l}1400,85 \\
(207,80)\end{array}$ & $\begin{array}{l}1216,05^{*} \\
(165,98)\end{array}$ \\
\hline $\begin{array}{l}\text { Pressão Plantar Média } \\
\qquad\left(\mathrm{g} / \mathrm{cm}^{2}\right)\end{array}$ & $\begin{array}{c}847,70 \\
(130,10)\end{array}$ & $\begin{array}{l}721,81^{*} \\
(43,37)\end{array}$ & $\begin{array}{c}976,05 \\
(219,07)\end{array}$ & $\begin{array}{l}888,94^{*} \\
(124,56)\end{array}$ & $\begin{array}{c}982,54 \\
(191,28)\end{array}$ & $\begin{array}{l}901,83^{*} \\
(125,30)\end{array}$ \\
\hline
\end{tabular}


* Diferenças significativas entre o grupo controle e o grupo fascite.

Na Tabela 2 foram apresentados os dados referentes as variáveis espaçotemporais para o grupo controle e para o grupo fascite plantar, na fase préintervenção e pós-intervenção.

Não foram encontrada diferenças estatisticamente significativas entre 0 GF e o GC para nenhuma das variáveis espaço-temporais. Também não foram encontradas diferenças significativas para o GF entre avaliação pré-intervenção e avaliação pós-intervenção.

Tabela 2 - Dados referentes as variáveis espaço-temporais.

\begin{tabular}{|c|c|c|c|c|c|c|}
\hline \multirow[b]{3}{*}{ Variável } & \multirow{2}{*}{\multicolumn{2}{|c|}{$\begin{array}{l}\text { Grupo Controle } \\
\text { (Média } \pm \text { DP) } \\
\text { Pré-Intervenção }\end{array}$}} & \multicolumn{4}{|c|}{$\begin{array}{c}\text { Grupos Fascite Plantar } \\
\text { (Média } \pm \text { DP) }\end{array}$} \\
\hline & & & \multicolumn{2}{|c|}{ Pré-Intervenção } & \multicolumn{2}{|c|}{ Pós-Intervenção } \\
\hline & Pé esquerdo & $\begin{array}{c}\text { Pé } \\
\text { direito }\end{array}$ & Pé esquerdo & Pé direito & Pé esquerdo & Pé direito \\
\hline Comprimento do passo $(\mathrm{cm})$ & $\begin{array}{l}58,45 \\
(3,22)\end{array}$ & $\begin{array}{l}59,53 \\
(3,79)\end{array}$ & $\begin{array}{l}58,82 \\
(6,59)\end{array}$ & $\begin{array}{l}59,62 \\
(5,48)\end{array}$ & $\begin{array}{l}59,55 \\
(3,66)\end{array}$ & $\begin{array}{l}59,53 \\
(3,79)\end{array}$ \\
\hline Cadência (passos/min) & $\begin{array}{l}53,95 \\
(5,61)\end{array}$ & $\begin{array}{l}54,04 \\
(6,94)\end{array}$ & $\begin{array}{l}55,28 \\
(6,77)\end{array}$ & $\begin{array}{l}55,25 \\
(6,74)\end{array}$ & $\begin{array}{l}53,80 \\
(6,61)\end{array}$ & $\begin{array}{l}54,04 \\
(6,94)\end{array}$ \\
\hline Largura do passo $(\mathrm{cm})$ & $\begin{array}{l}13,86 \\
(3,28)\end{array}$ & $\begin{array}{l}13,78 \\
(6,59)\end{array}$ & $\begin{array}{l}13,43 \\
(5,55)\end{array}$ & $\begin{array}{l}13,57 \\
(5,36)\end{array}$ & $\begin{array}{l}13,51 \\
(6,43)\end{array}$ & $\begin{array}{l}13,78 \\
(6,59)\end{array}$ \\
\hline Ângulo de progressão do pé( $\left(^{\circ}\right)$ & $\begin{array}{l}13,48 \\
(4,88)\end{array}$ & $\begin{array}{l}19,29 \\
(6,26)\end{array}$ & $\begin{array}{l}14,52 \\
(6,39)\end{array}$ & $\begin{array}{l}19,65 \\
(5,85)\end{array}$ & $\begin{array}{l}12,63 \\
(5,67)\end{array}$ & $\begin{array}{l}19,29 \\
(6,26)\end{array}$ \\
\hline Ângulo do eixo do pé $\left({ }^{\circ}\right)$ & $\begin{array}{l}12,63 \\
(6,85)\end{array}$ & $\begin{array}{l}13,23 \\
(4,66)\end{array}$ & $\begin{array}{r}13,34 \\
(3,93)\end{array}$ & $\begin{array}{l}42,53 \\
(6,93)\end{array}$ & $\begin{array}{l}12,62 \\
(6,04)\end{array}$ & $\begin{array}{l}13,23 \\
(4,66)\end{array}$ \\
\hline Velocidade $(\mathrm{cm} / \mathrm{s})$ & $\begin{array}{l}105,04 \\
(12,23)\end{array}$ & $\begin{array}{l}107,36 \\
(12,00)\end{array}$ & $\begin{array}{l}108,70 \\
(22,48)\end{array}$ & $\begin{array}{c}110,97 \\
(20,11)\end{array}$ & $\begin{array}{c}106,76 \\
(14,50)\end{array}$ & $\begin{array}{c}107,14 \\
(14,61)\end{array}$ \\
\hline
\end{tabular}

\section{DISCUSSÃO}

Conforme apresentado nos resultados, observou-se que os locais onde ocorreram os sintomas de dor foram no arco plantar e no calcanhar. Esses dados estão de acordo com Zanon et al. (2006) e Starkey e Ryan, 2001. Imamura et al. (1996) verificou que a fascite plantar é causa comum de dor na região do retropé. Com a palpação na região plantar observa-se o ponto gatilho na região medial e plantar da tuberosidade do calcâneo e a extensão dos dedos exacerba os sintomas pela distensão da fáscia plantar (GREVE e AMATUZZI, 1999). A presença de pontos dolorosos na porção proximal do músculo gastrocnêmio medial demonstra a concomitância de uma síndrome dolorosa miofascial que envolve o complexo calcâneo plantar (PERRY, 2005). 
Segundo os resultados, observa-se que o período do dia que ocorre os sintomas da dor com maior intensidade é no período da manhã. Estes dados estão de acordo com Williams e Wilkins (1996) que salientam que os sintomas de dor são mais evidentes pela manhã, ao dar o primeiro passo, ao se levantar da cama, ou após um período de repouso.

A partir dos resultados referentes à intensidade da dor (Figura 1), conforme a Escala de Dor Associada, observou-se que os sintomas da dor provocados pela fascite plantar reduziram após o uso da órtese (avaliação PósIntervenção). Estes dados corroboram com o estudo de Landorf et al. (2006), os quais relataram que os grupos de palmilhas pré-fabricadas e personalizada demonstraram benefícios a curto prazo, isto é, em 3 meses, e com Landorf et al. (2006) que relatam que a utilização temporária de uma órtese, seguido de alongamento dos tecidos moles foi bem sucedida na redução dos sintomas de dor nos pés com fascite plantar. $\mathrm{O}$ uso de órteses plantares tem sido orientado para redução das condições dolorosas relacionadas aos pés (HODGE et al., 1999). Sugere-se que as órteses podem afetar a distribuição das cargas plantares em contato com as superfícies rígidas, reduzindo a absorção de choques e, assim, poderiam diminuir as algias de membros inferiores e coluna lombar (SOBEL et al., 2001). De acordo com Alves et al. (2008), pacientes com fascite plantar apresentam pressão plantares maior na região do antepé sendo característico da patologia. Esse aumento da pressão plantar na região do antepé sugere que como a patologia ocasiona dor na região do retropé (calcanhar), o paciente acaba dotando uma postura antálgica como mecanismo compensatório para não comprometer ainda mais o local que se encontra acometido.

Foram observadas diferenças estatisticamente significativas entre o grupo GC e o GF para as variáveis pressão plantar máxima e pressão plantar média, para o pé direito (Tabela 1). No pé esquerdo não foram observadas diferenças significativas. A diferença nos resultados, entre pé esquerdo e direito, deve-se ao mecanismo compensatório, protegendo o local da dor, como pode ser observado nos resultados $70 \%$ dos participantes apresentam fascite plantar no pé direito, sendo que em somente um caso a fascite plantar era bilateral. Para as demais variáveis (tempo de contato e área de contato) não foram observadas diferenças significativas entre o GC e o GF. 
Para o GF não foram observadas diferenças significativas para as variáveis da distribuição da pressão plantar entre a avaliação pré-intervenção (uso da órtese) e pós-intervenção, mostrando que o uso da órtese não provocou alterações significativas no comportamento pressão plantar, tanto para o pé direto como para o pé esquedo.

Menz e Morris (2006) realizaram um estudo para correlacionar as alterações estruturais do pé e tornozelo com os valores de pressão plantar e observaram que os valores de pressão no mediopé e antepé estão associados ao índice de dor no arco plantar, a pressão na primeira articulação metatarso falangiana com a amplitude de movimento desta articulação, e, a pressão do hálux está associada com sua força flexora. Os autores colocam ainda que os resultados de testes clínicos realizados no pé e tornozelo podem explicar os valores de pressão plantar e estas informações podem ser úteis para o desenvolvimento de intervenções que amenizem as complicações relacionadas às descargas de pressão plantares inadequadas. Wearing et al. (2003 e 2007) colocam que os sintomas de dor nos pés de pacientes com fascite plantar promovem adaptações no padrão da marcha ocasionando uma redução das cargas no retropé e aumento das cargas nas regiões do mediopé, antepé e os dedos e que estas alterações ocorrem devido ao mecanismo de compensação para a prevenir os sintomas de dor. Sant'anna (2004) aborda que pacientes que apresentam fascite plantar tendem a possuir marcha antálgica, como apoio sobre a região lateral ou anterior do pé, sobre as cabeças dos metatarsos, assim não tendo impacto direto sobre o local doloroso.

Não foram encontrada diferenças significativas entre o GF e o GC para nenhuma das variáveis espaço-temporais (Tabela 2). Também não foram encontradas diferenças significativas para o GF entre avaliação pré-intervenção e avaliação pós-intervenção. Estes resultados não estão de acordo com Wearing et al. (2003 e 2007), que relata que o estímulo álgico nos pés de indivíduos com fascite plantar, durante a marcha, promove adaptações no padrão de rolamento do pé ocasionando uma redução da velocidade da marcha.

Os resultados encontrados na avaliação pós-intervenção, os quais não apresentaram diferenças significativas nas variáveis da distribuição da pressão plantar e espaço-temporais, podem ter sido influenciados pelo tempo de uso da órtese que foi muito reduzido, forma de confecção das órteses, a qual não 
corrigiu de forma adequada as alterações dinâmicas da marcha do paciente, a qualidade das informações adquiridas pelo sistema de distribuição de pressão plantar, problemas no momento da coleta dos dados que não foram controlados, tamanho da amostra do estudo e variabilidade das características dinâmicas da amostra.

\section{CONCLUSÕES}

Com base nos resultados observou-se que a fascite plantar provocou aumento da pressão plantar comparada com indivíduos sem fascite plantar, mas não apresentou alterações nas variáveis espaço-temporais.

O uso da órtese provocou uma melhora no quadro álgico dos pacientes com fascite plantar, porém não provocou alterações significativas no comportamento da distribuição da pressão plantar e das variáveis espaçotemporais.

Estes resultados podem ter sido influenciados pelo tempo reduzido de uso da órtese, forma de confecção das órteses, a qualidade das informações adquiridas pelo sistema de distribuição de pressão plantar, tamanho e variabilidade da amostra do estudo. A melhora no quadro álgico dos pacientes com fascite plantar pode ter sido influenciada pelo efeito placebo da órtese, sendo que a órtese provoca um apoio no arco longitudinal minimizando a tensão da fáscia plantar a qual pode ter contribuindo para a redução do quadro álgico durante a marcha.

Destaca-se a importância de novos estudo um maior número de participantes em diferentes faixas etárias e intervenções com tempo mínimo 3 meses de uso da órteses. 


\section{REFERÊNCIAS}

\section{ATENÇÃO DEVE CORRIGIR TODAS AS \\ REFERÊNCIAS DE ACORDO COM AS NORMAS DA REVISTA}

ALVAREZ, C.; DE VERA, M.; CHHINA, H.; BLACK A. Normative data for the dynamic pedobarography of children, Gait \& Posture, 2008.

ALVES, E.; LIMA, Z.B.; SEIXLACK, M.A.L.; BERTOLINE, G.R.F.; BUZANELLO, M.R. Avaliação da pressão plantar em indivíduos com fascite plantar. Revista Saúde e Pesquisa, v. 1, n. 3, p. 277-281, set./dez. 2008.

Bovi G' ${ }^{1}$ Rabuffetti M, Mazzoleni P, Ferrarin M. A multiple-task gait analysis approach: Kinematic, kinectic and EMG reference data for healthy young and adult subjects. Gait and Posture, 2011. Gait Posture. 2011 Jan;33(1):6-13.

BRICOTT, B. Posturologia. São Paulo: Ícone, 1999.

CARVALHO, D. S.; KOWACS, P. A. Avaliação de intensidade de dor. Rev Migrâneas Cefaléias. 9(4):164-168, 2006.

FERREIRA, R.C.; . revbras ortop.49(3):213-217. São Paulo, SP, Brasil. 2014.

GREVE, J. D. M.; AMATUZZI, M. M. Medicina de reabilitação aplicada à ortopedia e traumatologia. São Paulo: Roca, 1999.

HEBERT, S.; XAVIER, R. Ortopedia e traumatologia: princípios e prática. 3. ed. Porto Alegre: Artmed, 2003.

HODGE,M.C.; BACH,T.M. CARTER GM. Orthotic management of plantar pressure and pain in rheumatoid arthritis. Clin Biomech, 14(8): 567-75. 1999.

Imamura, M. et al. Fascite plantar: estudo comparativo. Revista Brasileira de Ortopedia. v. 31 , p. 561-566; $1996 . \quad$ Disponível em: 
<http://www.rbo.org.br/pdf/1996_jul_61.pdf>. Acesso em: 20/08/2012. NÃO ESTÁ CORRETO TEM VÁRIOS OUTROS AUTORES

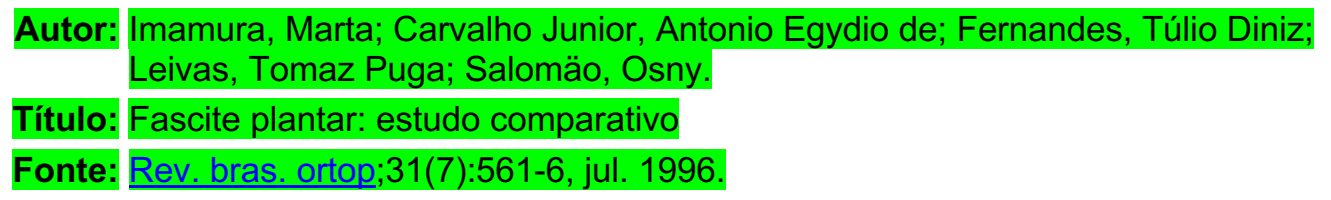

JANNINK,M.;VAN DIJK,H.;IJZERMAN,M.;GROOTHUIS-OUDSHOORN， K.; LANKHURST, J. G. Effectiveness of custom-made orthopedic shoes in the reduction of foot pain and pressure in patients with degenerative disorders of the foot. FootAnkle Int.; v. 27(11): p. 974-9. 2006

LANDORF,K.B.; KEENAN,A.M.; HERBERT,R.D. Orthotics not effective for plantar fasciitis. J Family Practice. 2006.

MANFIO, E. F.; MAYER, O. S.; AVILA, A. O. A. Avaliação pré e pós-operatório em crianças submetidas a tratamento cirúrgico mediante técnica de Cavalier. In: Congresso Brasileiro De Biomecânica, Anais, Santa Catarina. Florianópolis: UDESC, v.8, p.213-218, 1999.

MENZ HB, MORRIS ME. Clinical determinants of plantar forces and pressures during walking in older people. Gait \& Posture 2006; 24: 229-236.

http://dx.doi.org/10.1016/j.gaitpost.2005.09.002

METZKER, 2012). - Carlos Alexandre Batista Metzker A FRICÇÃO TRANSVERSA PROFUNDA NO TRATAMENTO DA FASCEÍTE PLANTAR CRÔNICA: ESTUDO DE CASO -

NÃO ESTAVA NA LISTA DE REFERÊNCIA (PARA AJUDAR COLOQUEI O TÍTULO DO TRABALHO

Mickle KJ, Munro BJ, Lord SR, Menz HB, Steele JR. Gait, balance and plantar pressures in older people with toe deformities. Gait Posture. 2011 Jul;34(3):34751 
MIYAZAKI, A; ANDRADE, M. PROATO, Programa de Atualização em Traumatologia e Ortopedia, ciclo 6 módulo 3. Porto Alegre,Artmed 2010. 258 p.

NAZARIO,P.F.; SANTOS,J. O. L.; AVILA,A. O. V.; Comparação da distribuição de pressão plantar em sujeitos com pés normais e com pés planos durante a marcha. Rev Bras Cineantropom Desempenho Hum 2010, 12(4):290-294.

NORDIN, M.; FRANKEL, V.H. Biomecânica Básica do Sistema Musculoesquelético. Rio de Janeiro: Guanabara Koogan S.A. p.194. 2003.

Orlin,Margo, and Thomas McPoil. 2000. Plantar Pressure Assessment. Physical Therapy 80 .

PERRY, J. Análise de Marcha. Barueri, SP: Manole. 368p. 2005.

ROSEBAUM, D.; BECKER H.P. Plantar pressure distribution measurements Tecnical Background and clinial applications. Foot and Ankle Surgery, v.14.1997.

SANT.ANNA, R. B. Tratamento da fascite plantar bilateral pela técnica da crochetagem: um estudo de caso. Monografia (Curso de pós graduação Lato Sendu -Fisioterapia em Traumatologia e Ortopedia). Universidade Estácio de Sá. Rio de Janeiro, 2004.

SOBEL, E.; LEVITZ, S.J.;CASELLI, M.A.; CHRISTOS, P.J.; ROSENBLUM, J. The effect of customized insoles on the reduction of postworkdiscomfort.JAmPodiatric. 2001.

STARKEY, C.; RYAN, J.L. Avaliação de lesões ortopédicas e esportivas. 1. ed. São Paulo, SP: Manole, 564 p. 2001.

STEBBINS, J. A.; HARRINGTON, M. E.; GIACOMOZZI, C.; THOMPSON, N.; ZAVATSKY, A.; THEOLOGIS, T. N. Assessment of sub-division of plantar pressure measurement in children. Gait Posture, v. 22, p. 372-6, 2005.

WEARING,S.C.; SMEATHERS,J.E.; URRY,S.R. The effect of plantar fasciitis on vertical foot-groundre action force. Clin Orthop Relat Res. 2003. 
WEARING, S.C.; SMEATHERS, J.E.; SULLIVAN, P.M.; YATES, B.; URRY, S.R.; DUBOIS, P.; Plantar Fasciitis: are pain and fascial thicknessassociated with arch shape and loading. PhysTher. 2007.

WILLIAMS; WILKINS. Textbook of clinical chiropractic: a specific biomechanical approach.Baltimore, EUA, 525 p. 1996.

ZANON, R.G.; BRASIL A.K.; IMAMURA, M. Ultra-som contínuo no tratamento da fasciíte plantar crônica. Acta Ortopédica Brasileira, v.14, n. 3, São Paulo 2006. Disponivel em: <http://www.scielo.br/pdf/aob/v14n3/a04v14n3.pdf>. Acesso em: 20/08/2012. 